\title{
New Bubble Mitomycin C Application Technique for Haze Prevention following Customized Photorefractive Keratectomy
}

C. KARAARSLAN*

World Eye Hospital, Adana, Turkey

Karaarslan: A novel technique for haze-free PRK

\begin{abstract}
Present investigation deals with a pilot study in which a new technique for applying mitomycin $C$ directly onto the ablated corneal surface in the form of an injector-administered bubble. Here, a technique was introduced and the observations made with this technique in this pilot study involving 125 patients who received wave front bilateral photorefractive keratectomy. Mitomycin $\mathrm{C}$ was administered using the common minisponge application technique in one eye and mitomycin $C$ was administered using the new bubble technique in the other eye. Photorefractive keratectomy presentation was compared between the minisponge-treated and bubble-treated eyes. Visual parameters such as visual acuity, spherical refractive error and cylindrical refractive error were similar in eyes across the two treatments before and after wave front photorefractive keratectomy. None of the minisponge-treated or bubble-treated eyes developed clinically evident photorefractive keratectomy haze. Compared to the minisponge-treated eyes, bubbletreated eyes showed faster healing ( $3 \mathrm{~d}$ vs. $5 \mathrm{~d}$ for contact lens bandage removal) and faster visual recovery ( $\leq 5 \mathrm{~d}$ vs. 7-10 d). In conclusion, the new bubble technique for applying mitomycin $\mathrm{C}$ after wave front photorefractive keratectomy provided a safe, effective and time-saving alternative to the standard mini sponge mitomycin $\mathrm{C}$ application technique.
\end{abstract}

Key words: Cornea, haze, mitomycin C, ocular surface, photorefractive keratectomy, refractive error

Photorefractive keratectomy (PRK) is a well-established ophthalmic procedure that was originally developed by Trokel et al., who performed the first reported laser surgery on a patient in $1987^{[1]}$. Subsequently, a series of improvements to the PRK procedure and equipment were made and PRK was approved for clinical ophthalmic use in the USA in $1996^{[1]}$. The goal of PRK is to correct refractive errors including myopia, hyperopia and astigmatism by reshaping the cornea to improve the focusing of light rays onto the retina ${ }^{[2,3]}$. Briefly, after preparation for wave front photorefractive keratectomy (PRK-Wt), a target section of Bowman's layer and the anterior stroma of the cornea is removed with an excimer laser, which produces far-ultraviolet light that can be used to remove tissue with precision through a non-thermal photochemical laser-tissue interaction ${ }^{[1,2]}$.

A notable potential complication of PRK-Wt is corneal haze, cloudiness on the operated cornea that can impair contrast sensitivity and visual acuity. The haze is thought to be produced during the corneal healing process by a conversion of keratocytes into myofibroblasts, which, 
compared to normal corneal tissue cells, are less transparent and associated with a more disorganized extracellular matrix that tends to scatter light ${ }^{[4,5]}$. The main risk factor for early haze development after PRK$\mathrm{Wt}$ is the removal of substantial tissue to correct high myopia ( $>6$ diopters) $)^{[6,7]}$. Additionally, patients are often advised to wear ultraviolet light-limiting sunglasses to prevent late-developing and worsening of haze ${ }^{[8,9]}$. Topical steroid drops may be prescribed to help reduce haze development. Moreover, it has become standard practice for ophthalmologists to use mitomycin $\mathrm{C}$ (MMC), a cytotoxic quinone produced by Streptomyces caespitosus best known for its antitumor activity, as a prophylactic treatment to prevent haze development by decreasing keratocyte density in the healing cornea, especially for patients whose eyes are subjected to ablation depths of $\geq 75 \mu \mathrm{m}^{[10,11]}$. In this clinical investigation, an attempt was made to explore whether the safety and efficacy of the MMC administration could be improved. Specifically, rather than laying MMC soaked minisponges on the eyes for periods of $\geq 12 \mathrm{~s}$, as is commonly done, a bubble of MMC solution was applied directly onto the ablated corneal surface and the bubble was expanded with balanced salt solution (BSS) until it bursts and the MMC solution could then be rinsed away easily and quickly with BSS. Here, these observations made in a pilot study involving a series of 125 patients who received bilateral PRK-Wt followed by MMC administered using the traditional sponge technique in one eye and the exploratory bubble technique in the other eye have been reported.

The subjects of this study were 125 young adult native Turkish patients ( 87 men and 38 women) seeking PRKWt at the World Eye Hospital to obviate the need for corrective lenses. A higher demand for PRK-Wt from men than women was noticed in Turkey due to the requirement for young people to complete military service, for which they should not have a flap scar. All 125 patients reported that they were seeking surgical vision correction in preparation for military service or for police service, both of which require uncorrected distance visual acuity (UDA) of at least $14 / 20$ in each eye without physical signs of ophthalmic surgery, such as the flap demarcation line left by Lasik procedures, corneal scars, or corneal deposits. These requirements resulted in PRK-Wt recommendation rather than Lasik or I-Lasik, for these patients.

The patients age ranged from 18 to $23 \mathrm{y}$ (mean age of $20.67 \pm 1.59 \mathrm{y}$ ). The range of preoperative central corneal thicknesses was 466-491 $\mu \mathrm{m}(478.0 \pm 37.7 \mu \mathrm{m})$. None of the patients had an ophthalmic or systemic disease. All the patients were informed about the purpose of the study and the differences between the sponge and bubble MMC application techniques. Participation was voluntary and all participants signed informed consent forms.

Prior to PRK-Wt, UDAs (converted from $\mathrm{X} / 20$ to decimal) ranged from 0.05 to 0.6 (same range in both eyes, with average UDAs of $0.287 \pm 0.146$ for the right eye and $0.292 \pm 0.141$ for the left eye. Prior to PRK-Wt, spherical refractive error values were in the range of +2.25 .to -3.50 dioptres, with a cylindrical refractive error in the range of 1.25 to -2.25 dioptres or without any cylindrical refractive error. The average preoperative spherical refractive error was $-1.83 \pm 0.66$ and $-1.17 \pm 0.87$ for cylindrical values. Preoperative UDA and refractive error values were similar between the left and right eyes (Student's t tests, $p>0.05$ ); each patient eyes were assigned randomly to the minisponge and bubble technique (one eye per technique).

Before PRK, the UDA of each eye was determined with a Snellen eye chart test and the refractive errors of each patient eyes were determined with an autokeratorefraktometer (KR 8900 Topcon, Tokyo, Japan). The refractive error data were used to program the excimer laser. Follow-up vision assessments were performed after 3-5 d (short-term follow-up) and after 20-22 d (long-term follow-up). PRK-Wt was performed as an outpatient operation. Before starting the operation on each eye, proparacaine hydrochloride drops $(0.5 \%$, Novartis) were applied to the eye for numbing. An eyelid holder was placed to prevent blinking (fig. 1A) and the patient was instructed to look at a blinking red fixation light to prevent eye movement. Then the outer layer of epithelial cells on the cornea was removed with a blade (crescent knife, BD Visitec). The cornea was reshaped with an excimer laser (Star S4 IR, Johnson \& Johnson Vision) that was pre-programmed with the customized measurements of the eye being operated on. The reshaping was performed by photo ablation of Bowman's layer and the anterior stroma.

To prevent the development of haze, MMC was applied to the freshly operated cornea in two ways, the traditional minisponge technique or the new bubble technique, with each patient having one eye (random laterality) subjected to each method of MMC application. For the traditional technique, minisponges were submerged in $0.02 \% \mathrm{MMC}$ and then applied topically to the ablated cornea for $13 \mathrm{~s}$. For the bubble technique, a bubble of $0.02 \%$ MMC $(450-555 \mu 1)$ was placed 


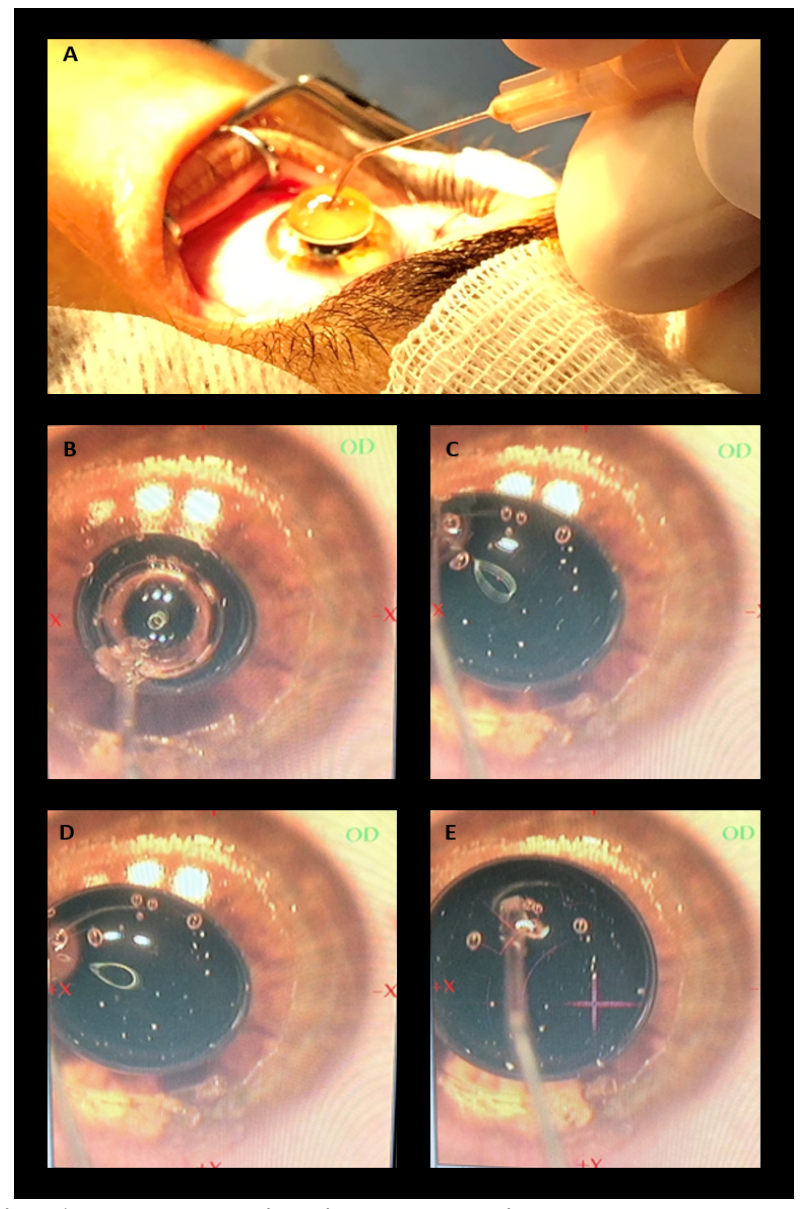

Fig. 1: Representative images showing the bubble MMC application technique

(A) Live photo of MMC bubble placed on a cornea immediately following PRK. (B-D) Series of images from a video recording of the technique showing progression of the MMC bubble as it is expanded over the photo ablated region in a tightly controlled manner.

onto the ablated corneal surface with a Pearce canula (25G $\times 7 / 8$ in, Beaver Visitec) creating a bubble (figs. 1A-B), which was expanded by intra-bubble filling (figs. 1C-E) via the same thin canula attached to a pre-filled syringe until the bubble covered the ablated area fully. Intra-bubble cannula application of BSS commenced immediately and progressed until the bubble burst. Immediately following removal of the minisponges or bursting of the bubble, the ocular surface was rinsed with BSS and a bandage contact lens was placed on the operated cornea to minimize discomfort during healing.

Patients were instructed to keep the bandage contact lenses on until it was determined that their corneas had healed sufficiently (3-4 d). For the postoperative re-epithelization period, patients were given steroid ophthalmic drops (dexamethasone $0.1 \%, 4 \times 1,10 \mathrm{~d}$ ) to limit inflammation, antibiotic ophthalmic drops (moxifloxacin $0.5 \%, 3 \times 1,10 \mathrm{~d}$ ) to prevent infection, and artificial tears (sodium hyaluronate $0.15 \%, 5 \times 1$, $3 \mathrm{mo}$ ) for lubrication. The adverse effect profile of moxifloxacin is much better with potent antibacterial property relative to the other agents, and hence moxifloxacin was opted over other fluoroquinolones. Based on visualization of healing, bandage contact lenses were removed $3 \mathrm{~d}$ postoperatively for eyes with bubble-applied MMC and $4 \mathrm{~d}$ postoperatively for eyes with minisponge-applied MMC.

Improvement in visual acuity was determined by UDA testing and post-PRK refraction error data was compared between eyes treated with the minisponge technique versus eyes with Student's t tests. Haze was assessed 3-5 d and 20-22 d after PRK-Wt with a bio microscope (Topcon). PRK was completed successfully in both eyes of all 125 patients included in this study without any perioperative adverse events or postoperative infectious keratitis. At the $3 \mathrm{w}$ postoperative follow-up, all 125 patients had a UDA of 1.0 (or better) in both individual eyes. As shown in fig. 2, at the $3 \mathrm{w}$ postoperative follow-up, neither spherical refractive error (Student's t test, $\mathrm{p}=0.0010$ ) nor cylindrical refractive error $(\mathrm{p}<0.0001)$ differed significantly between eyes subjected to the minisponge MMC administration technique and eyes subjected to the bubble MMC administration technique. None of the 125 eyes $(0 \%)$ treated with the bubble technique and none of the 125 eyes $(0 \%)$ treated with the minisponge technique showed signs of haze at the 3-5 d or 20-22 d follow-up examinations, and none of the 125 patients reported experiencing visual haze in their eyes at either follow-up examinations. At follow-ups conducted $3 \mathrm{mo}, 6 \mathrm{mo}$, and $12 \mathrm{mo}$ post-PRK, it was found that all the patients 250 eyes remained clear of haze.

In the present study, the utility of a new bubble technique

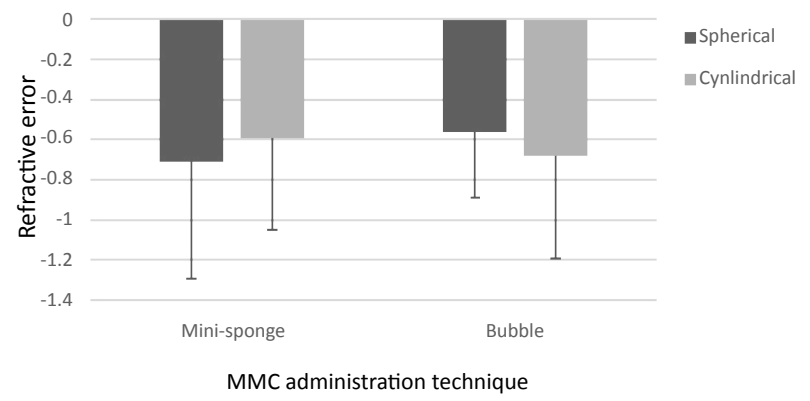

Fig. 2: Comparison of 3-week post-PRK mean refractive errors across MMC administration technique groups. Comparisons of the post-PRK spherical refractive errors $( \pm$ SD) observed with minisponge $(-0.71 \pm 0.58)$ versus bubble $(-0.56 \pm 0.33)$ application techniques and of the post-PRK cylindrical refractive error observed with minisponge $(-0.59 \pm 0.46)$ versus bubble ( $-0.68 \pm 0.51)$ techniques, ( $\square)$ spherical, $(\square)$ cylindrical 
for post-PRK application of MMC relative to the conventional minisponge MMC application technique was explored. With respect to outcomes, 20/20 or better (i.e. $\geq 1.0$ ) UDA was achieved in all 250 eyes, with no evident detrimental influence of the new bubble MMC technique relative to the minisponge MMC technique. None of the eyes subjected to either MMC application developed an early or a delayed persistent haze, suggesting that the new technique delivered MMC to the corneal surface effectively.

The new bubble technique of applying post-PRK MMC presented in this paper has become the favoured MMC application technique in the World Eye Hospital clinic owing to several associated advantages. During the procedure, the traditional minisponge technique required the use of minisponges, the preparation and application of which extended the procedural time. Although minisponge application of MMC is generally effective, it is unclear whether MMC held in minisponge material is being applied evenly over the corneal surface. Conversely, the new bubble technique obviated the need for use of minisponges and enabled MMC to be applied rapidly and evenly, with the operator in control of the contact interface area between the MMC bubble and the corneal surface.

Not only was the MMC application and rinse procedure with the bubble technique more time efficient than that with minisponge application, but also during this clinical experience it was found that patients' contact lens bandages could be removed a day earlier postoperatively owing to faster corneal epithelial healing. In general, it was found that the corneal epithelium was sufficiently healed within $4 \mathrm{~d}$ to allow bandage contact lens removal when MMC was applied using the bubble technique, as opposed to $5 \mathrm{~d}$ when the minisponge technique was applied. Moreover, thus far, all eyes that were subjected to the bubble technique in the present investigation have been clear of haze. In this context, it is important to note that direct application of MMC solution onto the eye, rather than onto an intermediary material such as minisponges, removed a potential risk of microbe transference to the cornea, which could lead to infectious keratitis, particularly that due to contamination with pathogenic Staphylococcus or Streptococcus species ${ }^{[12]}$. Finally, the more rapid healing seen in eyes treated with bubble-applied MMC, relative to eyes treated with minisponge-applied MMC, was followed by a shorter duration to full visual recovery. That is, at the $3 \mathrm{w}$ follow-up examination, patients reported experiencing full visual recovery within a week of the procedure in their bubble-treated eyes, with full visual recovery taking a few more days (i.e. $9-10$ d) in their minisponge-treated eyes.

The specific techniques used to deliver MMC following PRK are variable and evolving, particularly with respect to minimizing drug exposure. MMC exposure has been progressively reduced from $2 \mathrm{~min}$ to $12 \mathrm{~s}$ without reducing efficacy ${ }^{[13]}$. Additionally, although $\mathrm{MMC}$ is most commonly used at a concentration of $0.02 \%$, concentrations as low as $0.002 \%$ has been reported to prevent haze in eyes with shallow PRK ablation ${ }^{[14,15]}$. Given that MMC can disrupt cellular DNA, there are concerns regarding its ophthalmic safety. It is important to minimize damage to the corneal epithelium because corneal endothelial cells do not regenerate and the endothelial layer of the cornea functions to maintain tissue hydration and therefore clarity, of the cornea ${ }^{[16]}$. There has been inconsistency in the literature regarding the question of whether MMC reduces endothelial cell numbers in the cornea ${ }^{[17-19]}$. Thus, larger studies are needed to resolve whether and if so under what conditions MMC diminishes corneal endothelium. Ryan et al. compared wave front-guided versus wave frontoptimized PRK for visual performance and reported excellent and comparable results for visual and military performance at 6 mo follow-up ${ }^{[20]}$.

Given the aforementioned safety concern, the controlled application of MMC and immediate rinsing enabled by the bubble technique is noteworthy. Uncontrolled spreading of MMC over the anterior ocular tissues leaves the eye vulnerable to cytotoxic injury, especially if there is any delay or insufficiency in BSS irrigation following application of MMC. Using the bubble technique, MMC is applied in a singular operatorcontrolled bubble, whose expansion is stopped precisely upon abutting the edges of the circumference of the ablated surface area. Immediately thereafter, the MMC injector is replaced with the irrigation canula tip of an injector that was prefilled with BSS for irrigation. Hence, upon the MMC bubble reaching its target diameter, it is possible to insert the irrigation cannula and blow up the MMC bubble with BSS immediately, and then to continue to irrigate the entire anterior ocular surface with BSS within a couple seconds. During this procedure, ablated corneal tissue contact with the MMC solution bubble is $9-11 \mathrm{~s}$.

This pilot study had some limitations. Firstly, the sample population consisted entirely of healthy Turkish young adults. Thus, the generalizability of this observation to other populations is not known. Secondly, the sample 
included only 125 patients and 250 eyes. Procedural and outcome benefits of this bubble application technique should be examined in larger patient samples. In conclusion, these observations suggested that this new bubble technique for applying MMC after PRK provided a safe, effective, and time-saving alternative to the standard minisponge MMC application technique. The cohort of patients in this study, who were active young adults, exhibited faster healing and visual rehabilitation with bubble application of MMC than with minisponge application, enabling them to return to their normal routines without persistent visual disturbances. Larger studies encompassing a more heterogeneous sample are needed to verify whether this technique provided a reliable haze risk reduction.

\section{Medical Writing and/or Editorial Assistance:}

This manuscript was prepared with professional manuscript editing support from Dr. Ann Power Smith at Write Science Right.

\section{Authorship:}

The named author meets the International Committee of Medical Journal Editors (ICMJE) criteria for authorship for this article, takes responsibility for the integrity of the work as a whole, and has given his approval for this version to be published.

\section{Compliance with Ethics Guidelines:}

This article is based on previously conducted studies and does not contain any studies with human participants or animals performed by the author.

\section{Conflict of interest}

The authors declare that they have no conflict of interest.

\section{REFERENCES}

1. Trokel SL, Srinivasan R, Braren B. Excimer laser surgery of the cornea. Am J Ophthalmol 1983;96:710-15.

2. Corbett M, Maycock N, Rosen E, O'Brart D. Refractive Laser Surgery. In: Corneal Topography. Springer Cham: Switzerland. 2019;203-34.

3. Gomel N, Negari S, Frucht-Pery J, Wajnsztajn D, Strassman E, Solomon A. Predictive factors for efficacy and safety in refractive surgery for myopia. PLoS One 2018;13:e0208608.

4. Hashemi H, Miraftab M, Asgari S. Photorefractive keratectomy for mild-to-moderate myopics with thin corneas: A 3-year follow-up results. AJS 2017;4:2-5.

5. Alio J, Azar D. Management of Complications in Refractive Surgery. Springer, Cham. 2018;259-67.

6. Rosa N, De Bernardo M, Iaccarino S, Lanza M. Corneal biomechanical changes after myopic photorefractive keratectomy. Semin Ophthalmol 2015;30:328-34.

7. Ang BC, Foo RC, Lim EW, Tan MM, Nah GK, Thean LS, et al. Risk factors for early-onset corneal haze after photorefractive keratectomy in Asian population: Outcomes from the Singapore Armed Forces Corneal Refractive Programme 2006 to 2013. J Cataract Refract Surg 2016;42:710-16.

8. Stojanovic A, Nitter TA. Correlation between ultraviolet radiation level and the incidence of late onset corneal haze. J Cataract Refract Surg 2001;27:404-10.

9. Marino GK, Santhiago MR, Torricelli AA, Santhanam A, Wilson SE. Corneal molecular and cellular biology for the refractive surgeon: The critical role of the epithelial basement membrane. J Refract Surg 2016;32:118-25.

10. Teus MA, de Benito-Llopis L, Alio JL. Mitomycin C in corneal refractive surgery. Survey Ophthalmol 2009;54:487-502.

11. Margo JA, Munir WM. Corneal haze following refractive surgery: A review of pathophysiology, incidence, prevention, and treatment. Int Ophthalmol Clin 2016;56:111-25.

12. Donnenfeld ED, O'Brien TP, Solomon R, Perry HD, Speaker MG, Wittpenn J. Infectious keratitis after photorefractive keratectomy. Ophthalmology 2003;110:743-7.

13. Virasch VV, Majmudar PA, Epstein RJ, Vaidya NS, Dennis RF. Reduced application time for prophylactic mitomycin C in photorefractive keratectomy. Ophthalmology 2010;117:885-9.

14. Taneri S, Oehler S, MacRae S, Dick HB. Influence of a therapeutic soft contact lens on epithelial healing, visual recovery, haze, and pain after photorefractive keratectomy. Eye Contact Lens 2018;44:S38-43.

15. Naderi M, Sabour S, Khodakarim S, Daneshgar F. Studying the factors related to refractive error regression after PRK surgery. BMC Ophthalmol 2018;18:198.

16. Chen LY, Manche EE. Comparison of femtosecond and excimer laser platforms available for corneal refractive surgery. Curr Opin Ophthalmol 2016;27:316-22.

17. Gharaee H, Zarei-Ghanavati S, Alizadeh R, Abrishami M. Endothelial cell changes after photorefractive keratectomy with graded usage of mitomycin C. Int Ophthalmol 2018;38:121117.

18. Sánchez-González JM, López-Izquierdo I, Gargallo-Martínez B, De-Hita-Cantalejo C, Bautista-Llamas MJ. Bandage contact lens use after photorefractive keratectomy. J Cataract Refract Surg 2019;45:1183-90.

19. Morales AJ, Zadok D, Mora-Retana R. Intraoperative mitomycin and corneal endothelium after photorefractive keratectomy. Am J Ophthalmol 2006;142:400-4.

20. Ryan DS, Sia RK, Stutzman RD, Pasternak JF, Howard RS, Howell CL, et al. Wave front-guided versus wave frontoptimized photorefractive keratectomy: visual and military task performance. Mil Med 2017;182:e1636-44.

This is an open access article distributed under the terms of the Creative Commons Attribution-NonCommercial-ShareAlike 3.0 License, which allows others to remix, tweak, and build upon the work non-commercially, as long as the author is credited and the new creations are licensed under the identical terms

This article was originally published in a special issue, "Biomedical research applications in Pharmaceutical Sciences" Indian J Pharm Sci 2020:82(2)spl issue3;31-35 